\title{
Ambiguïtés, explicites et implicites dans les discours sur le métier de professeur documentaliste : quels enjeux collectifs pour la formation des élèves dans le second degré ?
}

Ambiguities, explicits and implicits in the speeches on the "professeur documentaliste" profession: what collective stakes for the education of pupils in secondary school?

Jocelyne Corbin Ménard

\section{CpenEdition}

\section{Journals}

Édition électronique

URL : http://journals.openedition.org/educationdidactique/2404

DOI : 10.4000/educationdidactique.2404

ISSN : 2111-4838

Éditeur

Presses universitaires de Rennes

\section{Édition imprimée}

Date de publication : 16 décembre 2015

Pagination : 127-141

ISBN : 978-2-7535-4878-7

ISSN : 1956-3485

Référence électronique

Jocelyne Corbin Ménard, « Ambiquïtés, explicites et implicites dans les discours sur le métier de professeur documentaliste : quels enjeux collectifs pour la formation des élèves dans le second degré ? », Éducation et didactique [En ligne], 9-3 | 2015, mis en ligne le 15 décembre 2017, consulté le 02 mai 2019. URL : http://journals.openedition.org/educationdidactique/2404 ; DOI : 10.4000/ educationdidactique.2404 


\title{
AMBIGUIITÉS, EXPLICITES ET IMPLICITES DANS LES DISCOURS SUR LE MÉTIER DE PROFESSEUR DOCUMENTALISTE: QUELS ENJEUX COLLECTIFS POUR LA FORMATION DES ÉLÈVES DANS LE SECOND DEGRÉ ?
}

\author{
Jocelyne Corbin Ménard \\ Laboratoire CREAD. Centre de Recherche sur l'Éducation, \\ les Apprentissages et la Didactique
}

\begin{abstract}
Lautonomie des établissements secondaires français pour mettre en œuvre les orientations de la politique éducative nationale implique que les acteurs s'approprient les prescriptions pour définir localement les objectifs communs au dispositif de formation qu'ils construisent. L'analyse de discours sur le métier de professeur documentaliste conduit à trois constats. D'abord, le Ministère de l'éducation nationale sur ses sites internet positionne ce professeur de manière ambigüe. Il le distingue voire l'exclut des personnels enseignants et mentionne ses domaines de compétences de façon plutôt floue. Ensuite, dans les organigrammes de collèges et de lycées publics, ce professeur est désigné et positionné diversement. Cette situation interroge sur la perception du métier et de sa fonction enseignante par les autres personnels. Enfin, différents groupes professionnels peinent à définir ce métier et ses spécificités. Outre les conséquences en termes de construction identitaire, se posent la question de la lisibilité de cette fonction et celle de la connaissance mutuelle des métiers comme condition nécessaire pour éviter des malentendus susceptibles de compromettre l'efficacité des réflexions et actions collectives.
\end{abstract}

Mots-clés: dispositif de formation, professionnalisation, établissement secondaire, identité professionnelle, action collective.

Ambiguities, explicits and implicits in the speeches on the "professeur documentaliste" profession: what collective stakes for the education of pupils in secondary school?

The autonomy of French secondary schools to implement the orientations of the national educational policy implies that stakeholders adapt the prescriptions in order to locally define common objectives for the teaching scheme they are building.

The speech analysis on the "professeur documentaliste " profession leads to three observations. Firstly, the ministry of national education gives an ambiguous place to this teacher on its websites. It distinguishes or even excludes her/him from the teaching staff and mentions her/his scopes of competence in a rather unclear manner. Then, in the secondary schools organization charts, this teacher is designated and positioned in various ways. This situation questions the perception of the profession and its teaching function by the other members of staff. Finally, different professional groups struggle to define this work and its specificities. Besides consequences in terms of identity construction, it challenges the readability of the function and of the mutual knowledge of the professions as a necessary condition to avoid misunderstandings likely to compromise the efficiency of the collective reflections and actions.

Keywords: teaching scheme, professionalization, secondary school, professional identity, collective action. 


\section{L'ÉTABLISSEMENT DU SECOND DEGRÉ, PORTEUR D'UN DISPOSITIF DE FORMATION}

Envisagé selon une approche systémique, l'Établissement Public Local d'Enseignement (EPLE) du second degré constitue un des niveaux de l'organisation du système éducatif français. À ce titre, les acteurs des collèges et lycées contribuent ensemble à poursuivre les finalités fixées par la politique éducative nationale. Or l'EPLE dispose d'une autonomie administrative et financière mais aussi pédagogique et éducative (Légifrance, 2014). Son efficacité est donc en partie tributaire de la manière dont les professionnels qui y exercent s'emparent des orientations ministérielles.

Porté par l'établissement, le « dispositif de formation », entendu comme "l'artefact fonctionnel qui matérialise une organisation particulière d'objets, d'acteurs, de structures et de systèmes de relations, en fonction des objectifs de formation dans une situation donnée » (Albero, 2010b, p. 51), se caractérise par une grande complexité. Il intègre plusieurs composantes que ses acteurs s'approprient et utilisent diversement dans la perspective de former les élèves: les prescriptions du ministère de l'Éducation nationale, des objets techniques physiques en différents lieux (CDI et autres salles) et des objets virtuels (tels que l'ENT), dotés de fonctionnalités potentielles. Cet ensemble structurel, avec l'organigramme des personnels et le "système de relations » (ibid.) qu'il sous-tend, configure l'établissement de manière singulière. Aussi, l'organisation du dispositif de formation résulte d'une dynamique portée par les choix des sujets grâce aux lignes des finalités qu'ils se fixent collectivement et individuellement au regard de ce qui est donné.

Cependant, la mise en œuvre effective dépend de l'équilibre entre trois dimensions interdépendantes et interagissantes qui, selon l'« approche trilogique des dispositifs en formation » (Albero, 2011), sont en tension permanente:

«l'idéel», qui oriente le travail selon des logiques d'action "épistémiques et axiologiques »;

«le fonctionnel de référence », qui oriente le travail selon des logiques d'action « fonctionnelles et instrumentales »

"le vécu», qui oriente le travail selon des logiques d'actions « intersubjectives et existentielles» (ibid.).
La ligne des finalités que les professionnels définissent relève de "la dimension idéelle » (ibid.) du dispositif. Elle est déterminée par les valeurs, les idéaux, les croyances, les aspirations des sujets. Lorsque cette dimension est prégnante, la prise en compte de la situation dans laquelle se déroule l'action risque d'être considérée de manière annexe. La ligne de la mise en ouvre de cet "idéel », des modalités et des techniques que les professionnels choisissent, préfèrent ou négocient avec leur environnement, relève du «fonctionnel de référence " en tant qu'opérationnalisation du projet depuis sa définition (règles, normes, organisation des lieux et des matériels, étapes de réalisation, etc.) jusqu'au fonctionnement effectif du dispositif (Albero, 2010a, 2010c, 2011). Cette dimension du dispositif de formation peut s'avérer dominante, par exemple si une trop grande importance est accordée aux aspects techniques et aux équipements technologiques par rapport aux actions mêmes d'éducation et de formation. La ligne des coopérations que les professionnels négocient en permanence dans la mise en œuvre de leur projet relève de « la dimension vécue » du dispositif dans la mesure où sont mis en jeu les manières de faire, les affects, les émotions des échanges intersubjectifs, mais aussi le sens que l'action conduite revêt pour chacun des acteurs (Albero, 2010a). Aussi, le développement de certaines affinités ou à l'inverse de certaines antipathies pourrait se faire au détriment de l'efficacité des coopérations indispensables à la conduite de l'action.

En somme, le bon fonctionnement du dispositif suppose un ajustement permanent de ces trois dimensions en tension structurelle. Si l'une ou l'autre est surinvestie, avec la perte de référence aux objectifs initiaux fixés dans le projet collectif ou celle de sa signification, c'est finalement l'efficacité du dispositif qui se trouve menacée. De même, l'opérationnalisation du projet dépend de la compréhension mutuelle de son ancrage. Aussi, les repères collectifs auxquels se réfèrent en premier lieu les acteurs du second degré doivent-ils être suffisamment précis, constants et convergents pour que les finalités définies ne reposent pas sur des malentendus.

Pourtant, l'étude de multiples discours véhiculés par le ministère de l'éducation nationale et par les professionnels à propos d'un des métiers de professeur du second degré, celui de "professeur documentaliste », fait apparaître des ambiguités quant à la désignation et au positionnement de celui-ci, 
auxquelles s'ajoutent des implicites dans les prescriptions et des écarts dans les représentations de ce métier (Corbin-Ménard, 2013). Cette situation, par la divergence de vues qu'elle reflète, interpelle le fonctionnement du dispositif de formation porté par l'établissement du second degré.

\section{DES AMBIGUIITÉS DANS LES DISCOURS MINISTÉRIELS ET PROFESSIONNELS}

Deux enquêtes menées dans une perspective comparatiste ont été conduites simultanément, de façon à se prémunir contre des variations de discours qui pourraient être imputables à l'évolution $\mathrm{du}$ contexte de politique éducative nationale. Elles se sont centrées sur des discours ministériels et sur ceux de professionnels du second degré concernant le professeur documentaliste. La première s'est appuyée sur des relevés effectués sur les deux principaux sites ministériels tandis que la seconde s'est référée à des organigrammes d'établissements publics consultables en ligne. Les deux enquêtes ont consisté en un premier recueil de données établi en novembre 2012, en l'espace de deux journées pour limiter les effets d'instabilité des ressources en ligne, puis en un second recueil constitué en janvier 2014 selon des modalités identiques, de manière à évaluer une éventuelle évolution des discours.

\section{Une désignation inconstante du professeur documentaliste}

Pour la première enquête, la méthodologie empruntée a cherché à garantir l'exhaustivité de l'investigation sur le site principal (http: //www. education.gouv.fr) et sur le portail national des professionnels de l'éducation Eduscol (http: // eduscol.education.fr), alimenté par la direction générale de l'enseignement scolaire (DGESCO) qui a la responsabilité de la fiabilité, de la validité et de la pertinence des informations déposées. Elle a recouru aux moteurs de recherche intégrés aux sites ministériels, sachant que sur le site principal, deux sortes d'outils sont proposés: l'un lance la recherche sur l'ensemble des pages Web, tandis que l'autre (Mentor) la restreint aux textes réglementaires parus au Bulletin Officiel (BO) ou au Journal Officiel (JO) depuis 1987 qui sont répertoriés, comme ceux du Ministère de l'enseignement supérieur et de la recherche, dans le Recueil des Lois et Règlements (RLR). Pour homogénéiser la démarche, les mêmes requêtes ont été soumises aux trois moteurs différents (les deux évoqués précédemment et celui du portail Eduscol). Elles portaient sur l'interrogation du seul mot « documentaliste », qui a aussi fait l'objet d'une requête dans sa variante au pluriel « documentalistes », car il s'est avéré que dans ce cas, des résultats différents étaient référencés. Chaque ressource signalée a ensuite fait l'objet d'une recherche au sein de son texte de l'une et l'autre forme du mot « documentaliste », afin que les extraits contenant ce mot soient identifiés puis éventuellement conservés. En revanche, les pages web secondaires accessibles par des liens pointant vers d'autres pages ou vers des dossiers n'ont pas été explorées, à l'exception d'un dossier intitulé « Portrait des enseignants de collèges et lycées: interrogation de 1000 enseignants du second degré en mai-juin 2004 » présentant a priori l'ensemble des enseignants du secondaire. Celui-ci a été sélectionné car il était en lien direct avec l'objet de l'étude, qui s'intéresse à l'organisation du second degré en général. L'objectif était plus précisément d'y rechercher ce qui était relaté à propos du professeur documentaliste et si une position ou une fonction spécifique lui était ou non attribuée. La sélection a exclu les ressources se rapportant à l'enseignement privé (organisé différemment, du fait de l'absence de CPE notamment), les illustrations de la mise en œuvre de la politique éducative (exemples ou expériences) et les résumés des débats ou communications effectués dans le cadre de colloques ou de séminaires. De même, les rapports n'ont pas été retenus car leurs préconisations ne sont pas toujours suivies d'injonctions ministérielles. Toutefois, les présentations de ces rapports et les résumés des articles de périodiques, accessibles de façon directe sur les pages web recensées, ont été conservés.

Pour la seconde enquête, la méthodologie n'a pas cherché à garantir l'exhaustivité de l'investigation, celle-ci étant au demeurant impossible. Il s'est agi en revanche d'employer une méthode de randomisation pour explorer les représentations de l'organisation du système que constitue l'établissement, telles qu'elles apparaissent sous forme d'organigrammes. Pour limiter le plus possible les problèmes d'instabilité des pages web, la recherche a été effectuée chaque fois en une même journée (le 20 novembre 2012 et le 18 janvier 2014). Elle a été réalisée au moyen d'un 
seul moteur de recherche, de manière à éliminer des variables qui pourraient influer sur les résultats des deux requêtes successives, en raison de la pluralité des méthodes de référencement et de la différence du nombre de pages indexées par les outils. Deux formulations ont été proposées successivement: " organigramme collège », puis « organigramme lycée » (sans guillemets). À la suite de chacune de ces requêtes, les cinq premières pages, contenant chacune dix résultats, ont été explorées. Seules ont été retenues les pages des établissements publics, quelle que soit la forme de la présentation (schéma ou une liste intégrant tout ou partie du personnel). $\mathrm{Au}$ total, un corpus de trente etun organigrammes d'établissement a pu être constitué (seize collèges et quinze lycées) en 2012 contre quarante-huit en 2014 (vingt-quatre collèges et vingt-quatre lycées).

De la première enquête centrée sur les discours, il ressort trois formulations différentes pour désigner le professeur documentaliste dans le discours véhiculé par le ministère de l'éducation nationale: « documentaliste », «professeur documentaliste » et « enseignant documentaliste ». Cette dernière désignation n'apparaît cependant qu'une seule fois, tant en 2012 qu'en 2014, dans la présentation d'une note d'information de la DEPP, portant sur l'éducation aux médias dans le second degré:

Les activités et supports proposés permettent de se familiariser avec les langages médiatiques et d'axer la réflexion sur ce thème de prédilection qu'est la liberté de la presse. Les enseignants documentalistes, les professeurs de français et d'histoire géographie sont les plus nombreux à s'impliquer dans l'éducation aux médias et, plus d'une fois sur deux, leur intervention au sein de la classe est conjointe (Ministère de l'Éducation nationale, 2009).

Des trois formulations, en 2012 comme en 2014, c'est le terme " documentaliste », au singulier ou au pluriel, utilisé seul, qui apparaît le plus souvent. Il est également usité dans les pages actualisées dans l'intervalle de temps.

De la seconde enquête centrée sur l'étude des organigrammes, il ressort une plus grande disparité encore dans les désignations. Aux trois formulations repérées sur les sites ministériels s'en ajoutent deux autres: « documentation» et «CDI». Ces disparités révèlent une importante confusion alors même que le corpus ne recense que quarante-huit organi- grammes (en 2014). En effet, lorsqu'il est mentionné et même nommé ( Monsieur X » ou « Madame $\mathrm{X} »$ ), ce professionnel est tantôt désigné par sa fonction, tantôt par le lieu dans lequel il exerce le plus souvent, tantôt par une pseudo-discipline $e^{1}$. De plus, dans certains cas, il est totalement absent en apparence mais il n'est pas possible de savoir alors s'il est inclus parmi les enseignants ou s'il a été oublié. Ces différentes terminologies (ou absence de mention) coexistent en collège et en lycée, tant en 2012 qu'en 2014. Les deux relevés effectués à quatorze mois d'intervalle font apparaître la prédominance de l'usage des termes « documentaliste » et «CDI», « documentaliste » restant plus fréquemment employé en collège, tandis que « CDI » continue d'être plus usité en lycée.

La pluralité des désignations perdure et prend même de l'ampleur puisqu'une nouvelle expression apparaît en 2014 dans les organigrammes : « enseignant documentaliste ». Si bien que l'usage d'un vocabulaire inapproprié pour désigner le « professeur documentaliste » demeure très présent.

Au total, le constat d'une disparité dans la désignation persiste, tant au plan du discours ministériel qu'au plan du discours de professionnels en charge d'élaborer et de valider les organigrammes rendus visibles par tous sur Internet. Cette situation invite à se demander si la fonction de ce professeur est clairement identifiée par le ministère de l'Éducation nationale et par ses personnels, d'autant qu'à cette confusion s'ajoute une pluralité de positionnements.

\section{Un positionnement du professeur documentaliste pluriel et contradictoire}

À partir de ces corpus de données recueillis sur les pages des sites ministériels et sur celles d'établissements, il apparaît que le positionnement attribué au professeur documentaliste par rapport aux autres personnels, et en particulier par rapport aux autres professeurs, est lui aussi inconstant. Dans le discours ministériel, ce professionnel est le plus souvent cité parmi d'autres: "Professeurs, documentalistes et conseillers principaux: cahier des charges de la formation $[\ldots]$ » (ministère de l'Éducation nationale, 2012j), parce qu'il est concerné par les mêmes prescriptions, mais il est aussi parfois mentionné isolément lorsqu'il s'agit de préciser son rôle spécifique: «Le professeur documentaliste forme les élèves à 
la recherche et à l'exploitation des informations. Il travaille en collaboration avec les enseignants et le conseiller d'orientation-psychologue. Il peut guider l'élève dans ses recherches d'informations ou de documentation » (ministère de l'Éducation nationale, 2010a). Dans le discours des professionnels du second degré, il figure tantôt parmi l'ensemble des personnels, tantôt parmi une partie seulement, lorsque les organigrammes ne sont pas exhaustifs. Pour autant, malgré les différentes circonstances de son évocation, quatre types de position sont identifiés dans les deux corpus constitués, et ce, aussi bien en 2012 qu'en 2014: la distinction, l'association ou l'intégration, l'exclusion par rapport au groupe des professeurs ou des enseignants.

La première position - la distinction - différencie ce professionnel d'autres personnels et des autres professeurs ou enseignants, par le biais d'énumérations, quelle que soit la manière dont il est désigné: «La formation des acteurs doit être organisée de façon anticipée pour répondre aux besoins de chacun: enseignant, chef d'établissement, conseiller principal d'éducation, conseiller d'orientation psychologue, professeur documentaliste » (ministère de l'Éducation nationale, 2011a). Il arrive aussi qu'il soit plus nettement séparé des professeurs du second degré, au même titre que les CPE, comme dans cette circulaire relative au diplôme national de master:

La réforme des conditions de recrutement et de la formation permettant de postuler aux métiers de l'enseignement - professeurs des premier et second degrés, professeur documentaliste et conseiller principal d'éducation (CPE) - doit permettre, dans le cadre notamment du cursus menant à l'obtention d'un diplôme national de master, d'améliorer la qualification des personnels, en vue de renforcer la réussite des élèves et de faciliter la mobilité au sein de l'Union européenne (Ministère de l'enseignement supérieur et de la recherche, 2010).

Dans les organigrammes, cette position se repère par le fait que soit mentionné un groupe désigné par " professeurs » ou « enseignants » auquel le professeur documentaliste n'est pas intégré.

La seconde position - l'association - correspond au fait que ces personnels sont désignés de façon distincte alors que leurs tâches paraissent être identiques. Les " documentalistes », sur cette page consacrée aux TPE, semblent être des personnels non enseignants associés à des enseignants: « Les documentalistes et les enseignants qui accompagnent ce travail, apprennent aux élèves à trouver des ressources et à les diversifier, ainsi qu'à sélectionner et analyser leur documentation » (ministère de l'Éducation nationale, 2013). Cette juxtaposition ne permet pas d'entrevoir ce qu'ils apportent de plus ou de différent aux élèves et invite même à se demander s'ils ne sont pas des "personnels supplémentaires » visant à soulager le travail de ceux qui sont désignés comme " enseignant » face à une classe entière.

Dans les organigrammes, ce cas de figure est matérialisé par une ligne reliant le professeur documentaliste aux professeurs ou aux enseignants, laissant sous-entendre qu'il s'agit d'une autre catégorie de personnel, même s'il est placé au même niveau sur la représentation de l'organisation de l'établissement.

Dans la troisième position - l'intégration - le professeur documentaliste, quelle que soit la manière dont il est désigné, fait partie tantôt du groupe des enseignants, tantôt de celui des professeurs, tantôt de celui des enseignants avec les professeurs, tantôt encore de l'équipe pédagogique, comme l'illustre l'extrait de cette page consacrée aux stages et au tutorat en lycée: « Les enseignants, professeurs documentalistes inclus, et les conseillers principaux d'éducation assurent le tutorat $»$ (ministère de l'Éducation nationale, 2012g).

Dans les organigrammes, cette position se traduit de trois manières: soit par la présence d'une mention spécifique (par exemple: "dont un documentaliste ») ; soit par le fait qu'il est mentionné au sein d'une liste de professeurs ou d'enseignants présentés selon leur discipline; soit par l'existence d'un lien hiérarchique le rattachant, comme les autres professeurs ou enseignants, à un "pôle pédagogique ».

La quatrième position - l'exclusion - correspond à l'octroi infondé au professeur documentaliste d'un statut différent de celui des autres professeurs. Ce cas de figure est présent dans la circulaire intitulée " diplôme national de master " destinée à cadrer les conditions de recrutement et de la formation permettant de postuler aux métiers de l'enseignement, où il est présenté comme un personnel passant un " concours » pour être recruté, alors que le "CAPES», cité parmi la liste des concours des professeurs du second degré, ne le concerne visiblement pas, bien qu'il soit lui aussi professeur certifié: «Concernant la préparation des concours, les spécificités propres aux concours de recrute- 
ment des professeurs des écoles, des CPE et des documentalistes, ainsi qu'au Capet, au CAPLP, au Capes et à l'Agrégation, doivent être prises en compte" (Ministère de l'enseignement supérieur et de la recherche, 2010). Une telle exclusion peut aussi être relative au groupe des enseignants auquel selon une étude de la DEPP réalisée dans les collèges publics en 2008-2009 à propos de la réussite de l'accompagnement éducatif au collège il ne semble pas appartenir, puisqu'il fait partie des « trois autres groupes » et plus précisément des « autres membres du personnel » qui ont apporté leur contribution:

Limplication de l'ensemble des intervenants

Les enseignants exerçant dans le collège ou non constituaient le groupe majoritaire d'intervenants dans tous les domaines d'activités de l'accompagnement éducatif, aux côtés de trois autres groupes:

les assistants d'éducation ou pédagogiques ;

les autres membres du personnel (documentalistes, conseillers principaux d'éducation, assistants de langue, etc.) ;

et les intervenants extérieurs au système éducatif. (ministère de l'Éducation nationale, 2011e)

Le constat de cette forme d'exclusion conduit à poser une autre question, celle de la relation de travail entre les différents personnels. Dans cette même étude un rôle subalterne est attribué au professeur documentaliste, car celui-ci est présenté comme l'exécutant d'une commande intervenu à la demande « du collège », c'est-à-dire de certains de ses personnels, plutôt que comme un contributeur de la co-construction d'un projet collectif: «Parmi les collèges participants de l'échantillon, un sur trois a fait intervenir le(s) conseiller(s) principal(-aux) d'éducation ou/et le(s) documentaliste(s) de l'établissement, par exemple » (ibid.).

L'exclusion est encore plus marquée dans une autre étude de la DEPP de 2004 qui dresse un « portrait des enseignants de collèges et lycées » au moyen de l'interrogation de 1000 enseignants du second degré. Cette enquête a pour but de répondre à trois questions: Qui sont les enseignants du second degré? Sont-ils satisfaits de leur métier? Dans quelle mesure sont-ils prêts à évoluer? L'échantillon est présenté comme étant représentatif (âge, sexe, statut, discipline, etc.). Pourtant, il ne comporte pas de professeurs documentalistes. Cependant, la question est posée aux différents professeurs de leur reconversion éventuelle. Si une petite majorité des enseignants du second degré souhaite rester dans l'éducation nationale, certains envisagent d'y exercer d' « autres fonctions », comme celle de « documentaliste », ce qui sous-entend qu'il s'agit d'une toute autre fonction que celle d'enseignant: "Au total, $57 \%$ souhaitent rester dans le système « éducation nationale » (contre $65 \%$ en 2002) dont $16 \%$ à l'université, les autres fonctions se répartissant entre chef d'établissement, IUFM, documentaliste ou conseiller en formation continue » (Ministère de l'éducation nationale, de l'enseignement supérieur et de la recherche, 2006).

Dans les organigrammes, l'exclusion se caractérise par le fait que ce personnel est mentionné alors que les groupes professionnels désignés par « enseignants », «professeurs » ou « équipe pédagogique » en sont absents. Dans environ un tiers des établissements (en 2012 et en 2014) et surtout en lycée, ce personnel figure ainsi parmi une liste d'autres professionnels non enseignants et se trouve même parfois inclus parmi le personnel administratif (une fois), parmi le personnel du service de la vie scolaire (une fois), parmi une liste mêlant des fonctions, des services ou des lieux de l'établissement (une fois) ou encore en 2014, parmi le personnel d'encadrement (une fois).

Au total, l'analyse des corpus ne conclut pas à une clarification des positions attribuées au professeur documentaliste, même si l'évolution de son positionnement dans le discours ministériel est marquée par l'introduction d'un nouveau « référentiel des compétences professionnelles des métiers du professorat et de l'éducation » (ministère de l'Éducation nationale, 2013) qui distingue pour la première fois des compétences spécifiques aux professeurs documentalistes tout en précisant qu'« outre les compétences qu'ils partagent avec l'ensemble des professeurs [...] énoncées ci-dessus, ils maîtrisent les compétences spécifiques ci-après " (ministère de l'Éducation nationale, 2013). En 2014, vingt-cinq pages web (contre vingtneuf en 2012) comportent encore des formulations ambiguës et contradictoires, certaines ayant disparu avec les quatre pages supprimées dans l'intervalle, tandis qu'une autre est apparue dans une nouvelle page consacrée à la lutte contre le décrochage scolaire, dans le sens où il a été jugé utile de préciser que le professeur documentaliste était un enseignant: « Le tutorat est un suivi personnalisé d'un élève ou 
d'un groupe d'élèves par un enseignant, dont les professeurs documentalistes, ou un conseiller principal d'éducation » (ministère de l'Éducation nationale, 2014). Parallèlement, l'hétérogénéité des positions du professeur documentaliste par rapport aux autres enseignants persiste dans les organigrammes en ligne en 2014. Si bien que l'identification de sa fonction enseignante par les auteurs de ces organigrammes et sa lisibilité pour les lecteurs de ces pages interpellent.

La multiplicité, l'imprécision et la divergence des désignations et des positionnements: un facteur de dysfonctionnement du dispositif de formation

Comme en 2012, les deux types de corpus comportent en 2014 une multiplicité de désignations et des divergences dans les positions attribuées. Cette persistance amène à se demander si la fonction enseignante du professeur documentaliste est finalement identifiable et identifiée par les autres acteurs. Aussi, dans le dispositif de formation, « ensemble organisé de moyens humains et matériels conçu, construit et mis en ouvre pour créer des conditions propices à des activités déterminées visant le développement des formés » (Corbin-Ménard, 2013, p. 390), s'il n'y a pas d'équivoque quant aux "formés » (les élèves), il n'en est pas de même quant aux "formants»: chacun sait par exemple que le professeur d'une discipline enseigne les rudiments de cette discipline à des élèves rassemblés dans des classes en se référant à un programme scolaire, mais la contribution du professeur documentaliste n'apparaît pas de manière évidente comme étant celle d'un enseignant. Car non seulement il est désigné par l'emploi indifférencié de différentes terminologies, mais intrinsèquement, certaines entretiennent une grande confusion, par exemple lorsqu'il est évoqué par son lieu de travail le plus fréquent (le CDI) plutôt que par sa fonction. Ce cas de figure s'observe assez souvent dans les organigrammes et constitue une particularité: il n'est pas commun de désigner les professeurs disciplinaires qui exercent dans des salles spécifiques (gymnase, salle de sciences, etc.) par leur lieu de travail. De fait, les différents discours traduisent, entretiennent, voire instaurent des repères bancals dans le cadre de référence du dispositif de formation porté par l'établissement du second degré car la «dimension idéelle » (Albero, 2010a, 2010b, 2010c, 2011) intègre des modèles conceptuels relatifs à l'implication de ce professeur dans la formation des élèves susceptibles d'entrer en contradiction.

Par ailleurs, les prescriptions primaires, c'est-àdire celles qui émanent de directives nationales, ne facilitent pas l'identification par les professionnels des champs de compétences et d'action du professeur documentaliste. En effet, les discours ministériels comportant peu d'explicites et beaucoup d'implicites, une large marge d'interprétations s'offre aux acteurs.

\section{EXPLICITES ET IMPLICITES DANS LES PRESCRIPTIONS PRIMAIRES ET DANS LE DISCOURS DES GROUPES PROFESSIONNELS}

\section{Dits et non-dits dans le discours institutionnel}

De nombreux domaines d'intervention du professeur documentaliste sont explicitement mentionnés dans les prescriptions nationales. Certains sont partagés avec les autres personnels: l'accompagnement personnalisé, le tutorat au lycée, l'aide aux élèves en difficulté, les travaux personnels encadrés, l'orientation, la découverte professionnelle en classe de troisième, l'évaluation de compétences du socle commun de connaissances et de compétences, etc. D'autres lui sont plus spécifiquement attribués depuis que ses missions ont été définies (ministère de l'Éducation nationale, 1986) : formation des élèves à la recherche et à l'exploitation d'informations sur l'orientation, formation des élèves à la recherche documentaire en général, gestion des ressources documentaires et informationnelles, responsabilité du centre de documentation et d'information, ouverture culturelle, etc. D'autres encore lui ont été assignés ou reformulés plus récemment: définition et mise en œuvre d'une politique documentaire, contribution à la définition du volet numérique de l'établissement, organisation des espaces de travail (espaces de ressources et d'information, salles d'études), contribution à la définition du programme d'action culturelle de l'établissement, mise en place de projets stimulant l'intérêt des élèves et la découverte, définition d'une stratégie pédagogique permettant à tous les élèves d'acquérir des compétences dans le domaine de l'éducation aux médias et à l'information, développement du travail autonome, etc. (ministère de l'Éducation nationale, de la jeunesse et de la vie 
associative, 2012 ; ministère de l'Éducation nationale. DGESCO, 2010 ; ministère de l'Éducation nationale, 2013). Aussi, les missions du professeur documentaliste (désigné comme « documentaliste-bibliothécaire » avant la création du CAPES de documentation en 1989) inscrivent son activité dans des perspectives collectives et dans des actions conjointes:

L'action du documentaliste-bibliothécaire est toujours étroitement liée à l'activité pédagogique de l'établissement [...] Il favorise l'initiation des élèves à la lecture des documents graphiques et audiovisuels et à l'utilisation de l'informatique, en liaison avec les professeurs dans le cadre des programmes, et, le cas échéant, en relation avec le foyer socio-éducatif [...] Il apporte son aide aux élèves en difficulté en travaillant en liaison avec les professeurs et les personnels d'éducation [...]. Il participe à l'ouverture culturelle de l'établissement [...]. Il entretient des relations avec des structures extérieures et avec d'autres établissements scolaires, etc. (ministère de l'Éducation nationale, 1986).

En 2010, il est rappelé qu'il agit dans un cadre pédagogique et à l'échelon global de l'établissement:

Il travaille en collaboration avec les professeurs de discipline [...]. Il coopère avec les personnels d'éducation pour mettre en place des situations éducatives et culturelles qui permettent le développement du travail autonome (ministère de l'Éducation nationale. DGESCO, 2010).

En précisant que son activité est « liée à l'activité pédagogique de l'établissement », est réalisée « en liaison avec...», implique des « relations avec » et un travail en « collaboration », le ministère de l'Éducation nationale positionne le professeur documentaliste à l'interface d'un système dans lequel il lui faut coopérer avec des acteurs internes et externes pour contribuer, par de multiples moyens, aux apprentissages et au développement des élèves.

Plus récemment, les compétences spécifiques qui lui ont été reconnues par le ministère de l'éducation nationale (2013) en plus des compétences qu'il partage avec l'ensemble des professeurs et des personnels d'éducation d'une part et avec les professeurs d'autre part (ibid.) soulignent davantage encore la nature collective de son travail et ses dimensions d'ingénierie: "définition de...», " mise en œuvre de...», « mise en place de... » et « organisation » sont des termes récurrents dans l'énoncé de ses compétences (ibid.). Si bien qu'au total, son activité est à la fois multifinalisée (Leplat, 1992, 1993 ; Goigoux, 2007) et multidirigée:

vers tous les élèves, elle est orientée par un objectif d'éducation, de développement ; vers tous les enseignants et les autres personnels, elle est orientée par un objectif de coopération, d'accompagnement ou de conseil ; vers tous les partenaires extérieurs potentiels, elle est orientée par un objectif de coopération.

Parce que son activité est référée à l'intégralité des élèves de l'établissement, via l'ensemble des partenaires internes et externes, la latitude dont il dispose pour adapter son action à la situation locale s'accompagne de nombreux implicites: l'étendue et la hauteur de son implication dans les différents domaines mentionnés dans les prescriptions, la part de son investissement dans chacun d'entre eux par rapport à celle des autres personnels, les destinataires de son action, les différentes temporalités de ses interventions, l'ensemble des modalités qu'il détermine pour la mise en œuvre, les repères qu'il se donne pour agir, etc. Ces non-dits ne facilitent pas la compréhension de son travail par les autres acteurs, d'autant que la multitude de cadres d'intervention potentiels et le caractère transdisciplinaire de sa fonction enseignante contrastent avec le travail du professeur du second degré chargé d'enseigner une discipline selon un emploi du temps déterminé, à une seule partie des élèves par année scolaire.

\section{Divergences dans les représentations du métier par différents groupes professionnels}

Les représentations du métier de professeur documentaliste que se font différents acteurs du second degré ont été recueillies quelques mois avant les données des premières enquêtes précitées, au moyen d'un questionnaire proposé au cours de l'année scolaire 2010-2011. Celui-ci comportait une seule question à compléter à volonté: «Le professeur documentaliste c'est celui qui...». Le choix de cette formulation a été motivé par l'intention de faciliter la spontanéité et par celle d'induire l'emploi consécutif d'un verbe. Il a été inspiré par la méthode d'association libre, employée aussi pour faire émerger les représentations sociales avec la recherche du « noyau central» (Abric, 2003). Par ce moyen, il s'agissait de recueillir des énoncés relatifs aux rôles, 
fonctions, activités ou tâches attribuées au professeur documentaliste.

L'échantillon se décomposait en quatre groupes professionnels, soit 205 personnes approchées à l'occasion de journées de formation: 68 professeurs documentalistes, 64 professeurs stagiaires non professeurs documentalistes, 62 conseillers principaux d'éducation, 11 personnels de direction. La passation a été effectuée sur les différents lieux en début de demi-journée pour limiter l'influence de la thématique de la formation et selon le même protocole: une brève présentation orale de la recherche par le chercheur lui-même et des précisions sur ce qui était entendu par "représentations du métier ». À tous, il a été dit qu'il ne s'agissait pas de se référer précisément au professeur documentaliste qui exerce dans leur établissement, mais à l'image qu'ils se font de ce métier en général, celle-ci étant cependant très probablement en partie construite à partir de leurs expériences personnelles. La composition des groupes d'enquêtés a donc été voulue comme aléatoire au niveau du sexe, du type d'établissement d'exercice, de l'ancienneté dans la fonction, le but étant de recueillir un large éventail d'énoncés, analysés ensuite indépendamment de ces variables, pour pouvoir confronter les représentations intergroupes et endogroupes (Deschamps, 1973; Doise, 1973).

Le corpus de données, composé au total de 1879 énoncés complétant la «question» a fait l'objet de quatre analyses successives: une analyse de discours visant à repérer, à la lecture, les champs lexicaux dominants ; une analyse de discours instrumentée par un logiciel d'analyse textuelle repérant les co-occurrences (Alceste 2012) ; une analyse de discours ciblée sur le choix des verbes suivant le mot « qui » au moyen du logiciel Sphinx Plus ${ }^{2}$ - Édition Lexica (V5) ; une analyse de contenu référée aux missions des professeurs documentalistes, telles qu'elles sont définies depuis 1986. De cette pluralité d'analyses ont émergé des points de convergence et des écarts intergroupes.

D’une façon générale, l'ensemble des professionnels évoque les domaines d'intervention (recherche documentaire, internet, médias, lecture), le cadre dans lequel elles se font, le type de relation qu'il entretient avec le professeur documentaliste (coopération, accueil, aide, conseil), les tâches qu'il lui semble accomplir, les modalités de son action (pilotage, gestion, formation, animation, recherche de la motivation). Toutefois, des écarts apparaissent dans le discours entre les différents groupes professionnels (tableau 1 ci-dessous). Les plus significatifs concernent le vocabulaire employé, celui-ci étant globalement nettement plus diversifié mais aussi plus centré dans deux domaines de leur activité (la formation des élèves ; l'information) chez les professeurs documentalistes que chez les autres professionnels. L'analyse des verbes montre notamment trois différences significatives:

la prégnance dans le groupe des professeurs documentalistes d'un vocabulaire centré sur l'acte d'enseigner, observable à partir de la spontanéité des réponses ; a contrario, la quasi-absence de ce même vocabulaire au profit du verbe "aider» dans les citations en premier rang des autres professionnels; la prédominance dans le groupe des professeurs documentalistes de la place de l'information, de la veille, la communication, l'exploitation et la formation des élèves; a contrario, la quasi-absence de cette dimension dans les autres groupes professionnels qui semblent ne pas associer spontanément le professeur documentaliste à un professionnel de l'information ; la prédominance chez les autres professionnels d'un vocabulaire reliant l'activité du professeur documentaliste à celle d'un gestionnaire de centre de ressources qui accueille un public. 
Tableau 1. Synthèse de l'analyse du discours centrée sur les verbes

\begin{tabular}{|c|c|c|}
\hline Groupes & $\begin{array}{l}\text { Groupe } \\
\text { " professeurs documentalistes " }\end{array}$ & $\begin{array}{l}\text { Groupe } \\
\text { "tous sauf professeurs } \\
\text { documentalistes" }\end{array}$ \\
\hline Diversité du vocabulaire & $\begin{array}{l}129 \text { verbes différents } \\
11,63 \text { citations en moyenne } \\
\text { par professionnel }\end{array}$ & $\begin{array}{l}119 \text { verbes différents } \\
6,87 \text { citations en moyenne } \\
\text { par professionnel }\end{array}$ \\
\hline $\begin{array}{l}\text { Prédominance Spontanéité } \\
\text { (d'après la citation de rang 1) }\end{array}$ & $\begin{array}{l}\text { former } \\
\text { apprendre } \\
\text { enseigner }\end{array}$ & aider \\
\hline $\begin{array}{l}\text { Prédominance globale } \\
\text { très significative } \\
\text { (d'après le calcul du khi } 2 \text { sur } \\
\text { l'ensemble des citations) }\end{array}$ & $\begin{array}{l}\text { communiquer } \\
\text { diffuser l'information } \\
\text { exercer de nombreuses tâches } \\
\text { exercer une veille } \\
\text { ouvrir au plan culturel }\end{array}$ & $\begin{array}{l}\text { aider } \\
\text { commander } \\
\text { encadrer } \\
\text { organiser }\end{array}$ \\
\hline $\begin{array}{l}\text { Prédominance significative } \\
\text { (d'après le calcul du khi } 2 \text { sur } \\
\text { l'ensemble des citations) }\end{array}$ & $\begin{array}{l}\text { élaborer } \\
\text { former } \\
\text { préserver le calme }\end{array}$ & $\begin{array}{l}\text { accueillir } \\
\text { gérer } \\
\text { fournir } \\
\text { prendre en charge } \\
\text { prêter }\end{array}$ \\
\hline
\end{tabular}

Les différents groupes professionnels n'attribuent pas le même poids (tableau 2 ci-dessous) à chacune de leurs missions. Les professeurs documentalistes soulignent davantage que les autres professionnels leur fonction et leur domaine de formation de façon explicite voire revendicative et le rôle qu'ils estiment jouer au niveau de l'ouverture culturelle et du développement du goût pour la lecture, tandis que les autres personnels (avec des nuances toutefois selon les groupes) semblent davantage percevoir leur fonction de gestionnaire d'un centre de ressources.
Parallèlement, les professeurs documentalistes semblent plus souvent inscrire leur action dans une logique collective à l'échelle de l'établissement (politique documentaire, veille documentaire, communication de l'information, pilotage, fonds documentaire dans son ensemble) que dans une juxtaposition d'actions plus isolées, plus ponctuelles, éparpillées et parcellaires davantage perçues par les autres professionnels (accueil, validation de compétences, prêt de documents, aide, etc.). 
Tableau 2. Écarts significatifs intergroupes an niveau du vocabulaire relatif à la prescription (ministère de l'Éducation nationale, 1986) ${ }^{2}$

\begin{tabular}{|c|c|c|c|}
\hline $\begin{array}{l}\text { Groupes } \\
\text { Axes } \\
\text { de la } \\
\text { circulaire }\end{array}$ & $\begin{array}{l}\text { Professeurs } \\
\text { documentalistes }\end{array}$ & Personnels de direction & $\begin{array}{l}\text { Personnels non } \\
\text { professeurs } \\
\text { documentalistes }\end{array}$ \\
\hline $\begin{array}{l}\text { Axe } 1 . \\
\text { Formation } \\
\text { recherche }\end{array}$ & $\begin{array}{l}\text { Culture de l'information }{ }^{1} \\
\text { Culture informationnelle } \\
\text { Info-documentaire } \\
\text { Education aux médias }\end{array}$ & & $\begin{array}{l}\text { Recherche } \\
\text { Document } \\
\text { Documentation } \\
\text { Exposé }\end{array}$ \\
\hline $\begin{array}{l}\text { Axe } 2 . \\
\text { Activité } \\
\text { pédagogique }\end{array}$ & $\begin{array}{l}\text { Politique documentaire } \\
\text { Motivation } \\
\text { Curiosité } \\
\text { Plaisir } \\
\text { Libre } \\
\text { Liberté } \\
\text { Pilotage } \\
\text { Projet }\end{array}$ & $\begin{array}{l}\text { Politique documentaire } \\
\text { Pilotage } \\
\text { Projet }\end{array}$ & $\begin{array}{l}\text { Accueil des élèves } \\
\text { Temps libre } \\
\text { " Pas cours » } \\
\text { Permanence } \\
\text { Validation des } \\
\text { compétences }\end{array}$ \\
\hline $\begin{array}{l}\text { Axe } 3 . \\
\text { Ouverture }\end{array}$ & $\begin{array}{l}\text { Veille documentaire } \\
\text { Veille informationnelle } \\
\text { Diffusion de l'information } \\
\text { Communication } \\
\text { Relations avec l'extérieur }\end{array}$ & & $\begin{array}{l}\text { Animation } \\
\text { Organisation d'expositions } \\
\text { Accompagnement de } \\
\text { sorties scolaires } \\
\text { Presse }\end{array}$ \\
\hline $\begin{array}{l}\text { Axe } 4 . \\
\text { Responsable } \\
\text { centre }\end{array}$ & $\begin{array}{l}\text { Fonds } \\
\text { CDI }\end{array}$ & & $\begin{array}{l}\text { Types de documents } \\
\text { Document } \\
\text { Documentation } \\
\text { Prêt } \\
\text { Emprunt }\end{array}$ \\
\hline $\begin{array}{l}\text { Axe } 5 . \\
\text { Introduction } \\
\text { synthèse }\end{array}$ & $\begin{array}{l}\text { Enseigne } \\
\text { Enseignant } \\
\text { Professeur }\end{array}$ & & $\begin{array}{l}\text { Aide } \\
\text { Accompagnement } \\
\text { Accompagne } \\
\text { Assiste }\end{array}$ \\
\hline
\end{tabular}

Au total, ces divergences questionnent sur la visibilité de son action et plus particulièrement sur la compréhension de sa fonction d'enseignant par l'ensemble des acteurs. Or celle-ci n'est ni facilitée par la définition ancienne des missions des professeurs documentalistes, ni par le fait que son action dirigée vers les élèves semble revêtir des formes indirectes très importantes (43\% environ des énoncés des professeurs documentalistes renvoie à des formes d'action indirecte ${ }^{3}$ contre $19 \%$ à des formes d'action directe $^{4}$ ). Par ailleurs, les écarts dans la terminologie employée laissent supposer des perceptions voire des conceptions différentes du métier. Par exemple, un ensemble d'expressions, absent de la « circulaire de missions » (ibid.), est employé uniquement par les professeurs documentalistes, qui paraissent ainsi interpréter ou actualiser ce qui est prescrit par l'appropriation progressive d'un vocabulaire issu des sciences de l'information et de la communication que les autres professionnels semblent méconnaître.

Enfin, les discours de certains professionnels stigmatisés parfois sur la comparaison des conditions de travail des professeurs documentalistes témoignent d'une méconnaissance de ce métier. Ce constat invite à penser qu'une grande partie du travail du professeur documentaliste n'est pas visible par les autres profes- 
sionnels et que les professeurs documentalistes en souffrent d'autant plus qu'ils estiment être surchargés de travail et n'ont pas les mêmes conditions d'exercice ni les mêmes droits que les autres professeurs (temps de pause, évolution de carrière, rémunération). À cela s'ajoute un sentiment de dépendance chez les professeurs documentalistes, vis-à-vis des autres professionnels ${ }^{5}$, car ils sont souvent tributaires de leur accord pour former les élèves. Celui-ci pourrait, avec les incompréhensions déjà évoquées, générer des tensions entre les différentes catégories de personnels exerçant dans les établissements du second degré susceptibles de retentir sur le fonctionnement du dispositif de formation.

\section{CONCLUSION}

Les enquêtes portant sur les pages des sites internet du ministère de l'Éducation nationale et d'établissements scolaires font ressortir des points communs entre ces deux sources de discours: une désignation inconstante, un positionnement pluriel et contradictoire du professeur documentaliste. Parallèlement, alors que les prescriptions primaires le concernant sont multiples et souvent implicites, les groupes professionnels non professeurs documentalistes semblent méconnaître les domaines de compétences et avoir peu conscience de la fonction enseignante de ce professeur. Ce métier souffre d'un défaut de lisibilité. Mal connu ou mal compris par tous, ses repères apparaissent brouillés, tant pour les différents groupes professionnels, qui en ont une vision parcellaire, que pour les professeurs documentalistes eux-mêmes, qui ne l'envisagent pas de manière consensuelle. Pourtant, l'activité du professeur documentaliste est censée se situer à l'interface des acteurs internes et externes à l'établissement et être dirigée vers les apprentissages et le développement de tous les élèves. Cette situation peut conduire les différents acteurs à vivre leur investissement dans le métier sur un mode d'autant plus dissonant que les prescriptions qui engagent le professeur documentaliste à agir collectivement ne trouvent pas toujours un écho explicite adressé aux autres personnels. En effet, le "référentiel des compétences des métiers du professorat et de l'éducation» (2013) attribue aux professeurs documentalistes des responsabilités impliquant des coopérations dans différents domaines (dont l'éducation aux médias et à l'information et la lecture) :

"Les professeurs documentalistes, enseignants et maîtres d'œuvre de l'acquisition par tous les élèves d'une culture de l'information et des médias ». Pour cela, ils doivent apporter « les aides nécessaires aux élèves et professeurs [...] pour que les apprentissages et l'enseignement prennent en compte l'éducation aux médias et à l'information » et " définir une stratégie pédagogique permettant la mise en place des objectifs et des apprentissages » $[\ldots]$ « en concertation avec les autres professeurs »; " Les professeurs documentalistes, acteurs de l'ouverture de l'établissement sur son environnement éducatif, culturel et professionnel » doivent « développer une politique de lecture en relation avec les professeurs » (ibid.).

Pourtant, ce texte n'évoque ni l'éducation aux médias ni le développement d'une politique de lecture parmi les compétences des professeurs ou d'autres personnels et ne mentionne pas non plus de coopération avec les professeurs documentalistes dans ces domaines, ni dans d'autres d'ailleurs. Cette absence de réciprocité interroge sur l'appropriation d'un « fonctionnel de référence » commun à tous les acteurs de l'établissement.

Au bilan, il apparaît finalement que le système éducatif français s'est doté d'une organisation intégrant un professionnel, le professeur documentaliste, dont les apports et les atouts sont mal identifiés, peut-être en partie parce que sa fonction enseignante se démarque du modèle prégnant de professeur que connaît le second degré, principalement fondé sur la transmission de contenus disciplinaires.

Aussi, cette étude amène à considérer d'autres enjeux.

En premier lieu, l'étude encourage à réviser la définition de la fonction enseignante, en questionnant les liens entre la construction de l'identité professionnelle, telle que les différents acteurs se l'approprient et la diversité des formes d'activité, telles qu'elles se pratiquent, pour assumer cette fonction dirigée vers le développement de tous les élèves du second degré.

En second lieu, l'étude invite à questionner les actions conduites individuellement et collectivement à l'échelon d'un établissement pour permettre l'acquisition, par tous les élèves, de certains savoirs dits « transversaux » (l'éducation aux médias et à l'infor- 
mation entre autres), afin d'évaluer précisément l'efficacité des dispositifs de formation portés par les établissements du second degré dans ce domaine et dans d'autres. Au-delà, c'est la cohérence du dispositif de formation qu'il importe de comprendre. $\mathrm{Au}$ vu des résultats de la présente étude, on peut s'interroger sur les effets des "logiques d'action» sous-tendues par les trois dimensions du dispositif (Albero, 2011) : les divergences dans les conceptions (logique épistémique) et les finalités (logique axiologique) ne sont-elles pas susceptibles d'entrer en contradiction voire en conflit avec diverses formes possibles d'organisation ingénierique, logistique et technique (logique instrumentale) ?

En troisième lieu, c'est le processus d'appropriation de différents repères collectifs qui pourrait être étudié. L'étude montre que les conditions d'une bonne articulation entre politique nationale et adaptation locale ne semblent pas toutes réunies. En effet, comme le souligne Clerc (2011), « les collèges et les lycées doivent recevoir une commande claire, avec des indicateurs pour le pilotage et le contrôle de leurs actions ». Mais au-delà de la « commande », l'efficacité du dispositif de formation repose aussi sur un autre repère collectif: celui du métier de chacun. Or, d'un côté, pour l'enseignant du second degré, « l'instance transpersonnelle » (Clot, 2005) construite sur la base d'une mémoire collective servant de référence au sujet, est fragilisée (ibid.) ; de l'autre, pour les professeurs documentalistes, cette instance n'est ni consolidée par des règles génériques ni étayée par la mémoire collective, du fait du caractère encore récent du métier. Parallèlement, des changements importants viennent perturber les repères précédemment construits: l'émergence du numérique, la génération d'élèves « Digital Natives » (Prensky, 2001), le fort renouvellement du corps enseignant, les modalités d'évaluation des élèves par compétences, etc. Ils nécessitent une évolution de l'organisation du travail axée sur une action plus collective, dont la définition d'objectifs communs de formation fait partie. La construction et la mise en ouvre du dispositif de formation doivent pouvoir s'appuyer sur le ciment de la connaissance mutuelle des métiers, de façon à ce que les non-dits, les implicites et les sous-entendus des prescriptions, constituent des espaces de création que les acteurs puissent investir pour décliner et adapter la politique nationale au niveau local, et non des obstacles. Car dans ces métiers adressés à autrui, au moins trois enjeux collectifs sont interdépendants: le développement professionnel, qui résulte du croisement de l'acquisition de compétences professionnelles et de la dynamique de l'identité professionnelle au niveau individuel et collectif (Piot, 2009) ; le processus de professionnalisation des acteurs qui «s'alimente et se nourrit du croisement de trois projets: institutionnel, collectif et personnel » (Kaddouri, 2005, p. 147) ; le service rendu par le dispositif de formation considéré dans sa globalité. Même si chaque sujet a « pour intention première de rechercher une meilleure efficacité de son action, dans la situation où il est placé » (Wittorski, 2005, p. 212), son action, pour être efficace, suppose également que soit optimisée une action collective levant les malentendus sur le positionnement de chacun, au risque d'un fonctionnement soumis à de nombreux compromis... voire d'un fonctionnement du dispositif de formation compromis.

\section{NOTES}

1. L'expression "pseudo-discipline " est utilisée pour spécifier qu'il n'existe pas de discipline scolaire propre, ni de contours précis à la terminologie polysémique « documentation ».

2. Les termes en gras ne figurent pas dans la circulaire des missions. Ils signalent les écarts les plus significatifs.

3. Les énoncés sont classés dans la catégorie « action indirecte " pour les élèves lorsque sans équivoque, et sans qu'il soit besoin d'en savoir plus sur la situation, ce qui est dit par les enquêtés renvoie à des activités menées en dehors de la présence des élèves concernés, ou n'a pas de lien direct avec l'activité des élèves présents dans le même le lieu que le professeur documentaliste (le CDI, le plus souvent).

4. Les énoncés sont classés dans la catégorie «action directe » auprès des élèves lorsque sans équivoque, et sans qu'il soit besoin d'en savoir plus sur la situation, ce qui est dit par les enquêtés correspond à une intervention adressée directement aux élèves en leur présence.Dans la catégorie « action directe et / ou indirecte », sont classés les énoncés qui renvoient à des actions dont les modalités peuvent être de nature directe et indirecte à la fois, ou de nature directe ou indirecte selon les cas, selon les situations, ces dernières n'étant pas identifiables à la lecture des énoncés.

5. " a son travail trop dépendant de la représentation qu'en a le chef d'établissement "; " est dépendant du bon vouloir des collègues». 


\section{RÉFÉRENCES}

Abric, J.-C. (2003). La recherche du noyau central et de la zone muette des représentations sociales. Dans J.C. Abric (dir.), Méthodes d'étude des représentations sociales (p. 59-80). Ramonville Saint-Agne, France: Erès.

Albero, B. (2010a). De l'idéel au vécu : le dispositif confronté à ses pratiques. Dans N. Poteaux \& B. Albero (dir.), Enjeux et dilemmes de l'autonomie. Une expérience d'autoformation à l'université. Étude de cas (p. 67-94). Paris, France: Maison des sciences de l'homme.

Albero, B. (2010b). La formation en tant que dispositif\& du terme au concept. Dans B. Charlier \& F. Henri (dir.), Apprendre avec les technologies (p. 47-59). Paris, France: Presses universitaires de France.

Albero, B. (2010c). Une approche sociotechnique des environnements de formation: rationalités, modèles et principes d'action. Éducation \& Didactique, 4(1), 7-24.

Albero, B. (2011, juin). Une approche trilogique des dispositifs en formation: pourquoi est-ce que les choses ne fonctionnent-elles jamais comme prévu? Communication présentée au colloque OUFOREP, Nantes.

Clerc, F. (2011). Laccompagnement: vecteur de transformation de l'école? Cahiers pédagogiques, (HS 22). Repéré à http://www.cahiers-pedagogiques.com/Aideret-accompagner-les-eleves-dans-et-hors-l-ecole-7036

Clot, Y. (dir.). (2005). Enjeux du travail et « genres » professionnels dans la recomposition en cours des métiers de la fonction publique. Le cas des professeurs de l'enseignement secondaireetdupersonnel soignantdegérontologie. Repéréà http://potoscam.free.fr/Travail\%20Jenny\%20Mieze/ rapport\%20stage\%20exemple/Clot\%5B1\%5D.pdf

Corbin-Ménard, J. (2013). La construction d'un métier de l'enseignement entre logiques identitaires et activité des sujets. Le travail du professeur documentaliste: une conception contemporaine de la fonction enseignante? (Thèse de doctorat). Université de Rennes 2. Repéré à http://tel.archives-ouvertes.fr/docs/00/93/59/94/PDF/2 013theseCorbinMenardJpartielle.pdf

Deschamps, J.-C. (1973). L'attribution, la catégorisation sociale et les représentations intergroupes. Bulletin de psychologie, 13-14, 710-721.

Doise, W. (1973). Relations et représentations intergroupes. Dans S. Moscovici (dir.), Introduction à la psychologie sociale (p. 195-214). Paris, France: Larousse.

Goigoux, R. (2007). Un modèle d'analyse de l'activité des enseignants. Éducation E Didactique, 1(3), 47-70.

Kaddouri, M. (2005). Professionnalisation et dynamiques identitaires. Dans M. Sorel \& R. Wittorski (dir.), La professionnalisation en actes et en questions (p. 145-158). Paris, France: L'Harmattan.

Leplat, J. (1992). Lanalyse du travail en psychologie ergonomique: recueil de textes, 1 . Toulouse, France: Octarès.

Leplat, J. (1993). Lanalyse du travail en psychologie ergonomique: recueil de textes, 2 . Toulouse, France: Octarès.

Ministère de l'Éducation nationale. (1986). Missions des personnels exerçant dans les centres de documentation et d'information (Circulaire $\mathrm{n}^{\circ}$ 86-123 du 13 mars
1986). Repéré à http://www.cndp.fr/savoirscdi/index. php?id=209

Ministère de l'Éducation nationale. (2009). Léducation aux médias dans le second degré. Repéré à http://www.education.gouv.fr/cid23163/l-education-aux-medias-dansle-second-degre.html

Ministère de l'Éducation nationale. (2010). Acteurs de l'orientation. La communauté éducative et les partenaires extérieurs. Repéré à http://eduscol.education.fr/ cid47375/communaute-educative-et-partenaires.html

Ministère de l'Éducation nationale. (2011a). 15 repères pour la mise en ouvre. Repère $n^{\circ} 15$. Formation des acteurs. Repéré à http://eduscol.education.fr/cid49547/ formation-des-acteurs.html

Ministère de l'Éducation nationale. (2011b). Réussir l'accompagnement éducatif au collège. Extraits de l'étude de la DEPP. Repéré à http://eduscol.education.fr/cid56695/ extraits-de-l-etude-de-la-depp.html

Ministère de l'Éducation nationale. (2012a). Les dispositifs d'accompagnement des lycéens. Repéré à http://www. education.gouv.fr/cid48652/dispositifs-d-accompagnement-des-lyceens.html

Ministère de l'Éducation nationale. (2012b). Professeurs, documentalistes et conseillers principaux d'éducation. Cahier des charges de la formation. Repéré à http:// www.education.gouv.fr/pid25535/bulletin_officiel. html?cid_bo=60170

Ministère de l'Éducation nationale. (2013). Travaux personnels encadrés - TPE mode d'emploi. Repéré à http:// eduscol.education.fr/cid48137/tpe-mode-d-emploi. html

Ministère de l'Éducation nationale. (2013). Référentiel des compétences professionnelles des métiers du professorat et de l'éducation. Bulletin officiel, (30). Repéré à http://www.education.gouv.fr/pid25535/bulletin_officiel.html?cid_bo=73066

Ministère de l'Éducation nationale. (2014). La lutte contre le décrochage scolaire. Repéré à http://www.education. gouv.fr/cid55632/la-lutte-contre-le-decrochage-scolaire.html

Ministère de l'Éducation nationale, de l'enseignement supérieur et de la recherche. (2006). Portrait des enseignants de collèges et de lycées: interrogation de 1000 enseignants du second degré en mai-juin 2004. Repéré à http://media.education.gouv.fr/file/50/4/2504.pdf

Ministère de l'Éducation nationale, de la jeunesse et de la vie associative. (2012). Vademecum: vers des centres de connaissances et de culture. Repéré à http://cache. media.eduscol.education.fr/file/Innovation_experimentation/58/7/2012_vademecum_culture_int_ web_214771_215587.pdf

Ministère de l'Éducation nationale. DGESCO. (2010). Repères pour la mise en ouvre du Parcours de formation à la culture de l'information. Repéré à http://eduscol.education.fr/cid53581/reperes-pour-mise-amp-oelig-uvreparcours-formation-culture-information.html

Ministère de l'Enseignement supérieur et de la recherche. (2010). Diplôme national de master. Repéré à http:// 
www.enseignementsup-recherche.gouv.fr/pid20536/ rubrique-bo.html?cid_bo=50134

Légifrance. (2014). Organisation et fonctionnement des établissements publics locaux d'enseignement. Repéré à http://www.legifrance.gouv.fr/affichCode.do; jsessioni $\mathrm{d}=8$ DD8B93F34C803E773AC1E5EC529323C.tpdjo0 7v_1?idSectionTA=LEGISCTA000006166622\&cidTe xte=LEGITEXT000006071191\&dateTexte $=20090429$

Piot, T. (2009). Quels indicateurs pour mesurer le développement professionnel dans les métiers adressés à autrui? Questions vives, 5(11), 259-275. doi: 10.4000/ questionsvives. 622
Prensky, M. (2001). Digital natives, digital immigrants. On the Horizon. MCB University Press, 9(5). Repéré à http://www.employmentandlaborinsider.com/wpcontent/uploads/sites/328/2015/05/5.7.15.Prensky-Digital-Natives-Digital-Immigrants-Part 1.pdf

Wittorski, R. (2005). Les dynamiques de professionnalisation des individus, des activités et des organisations. Dans M. Sorel \& R. Wittorski (dir.), La professionnalisation en actes et en questions (p. 211-243). Paris, France: LHarmattan. 